\title{
Utilization of inhaled nitric oxide after surgical repair of truncus arteriosus: A multicenter analysis
}

\author{
Christine M. Riley MSN, APRN ${ }^{1}$ (D) | Christopher W. Mastropietro MD \\ Peter Sassalos MD ${ }^{3}$ | Jason R. Buckley MD ${ }^{4}$ | John M. Costello MD, MPH $^{4}$ | \\ Ilias Iliopoulos MD ${ }^{5}$ | Aimee Jennings MSN, APRN ${ }^{6}$ | Katherine Cashen DO \\ Sukumar Suguna Narasimhulu MD ${ }^{8}$ | Keshava M. N. Gowda MBBS ${ }^{9}$ \\ Arthur J. Smerling MD ${ }^{10}$ | Michael Wilhelm MD ${ }^{11}$ | Aditya Badheka MBBS ${ }^{12}$ | \\ Adnan Bakar MD ${ }^{13,14}$ | Elizabeth A. S. Moser MS ${ }^{15}$ | Venu Amula MD ${ }^{16}$ Collaborative \\ Research in Pediatric Cardiac Intensive Care (CoRe-PCIC) Investigators
}

${ }^{1}$ Department of Pediatrics, Division of Cardiac Critical Care, Children's National Health System, Washington, District of Columbia
${ }^{2}$ Department of Pediatrics, Division of Critical Care, Indiana University School of Medicine, Riley Hospital for Children, Indianapolis, Indiana
${ }^{3}$ Department of Cardiac Surgery, Section of Pediatric Cardiovascular Surgery, University of Michigan, C.S. Mott Children's Hospital, Ann Arbor, Michigan
${ }^{4}$ Department of Pediatrics, Division of Cardiology, Medical University of South Carolina Children's Hospital, Charleston, South Carolina
${ }^{5}$ Department of Pediatrics, Division of Cardiac Critical Care, The Heart Institute, Cincinnati Children's Hospital Medical Center, Cincinnati, Ohio
${ }^{6}$ Department of Pediatrics, Division of Critical Care, Seattle Children's Hospital, Seattle, Washington
${ }^{7}$ Department of Pediatrics, Division of Critical Care, Wayne State University School of Medicine, Children's Hospital of Michigan, Detroit, Michigan
${ }^{8}$ Department of Pediatrics, Division of Cardiac Intensive Care, University of Central Florida College of Medicine, The Heart Center at Arnold Palmer Hospital
for Children, Orlando, Florida
${ }^{9}$ Department of Pediatrics, Division of Critical Care Medicine, Cleveland Clinic, Cleveland, Ohio
${ }^{10}$ Department of Pediatrics, Division of Critical Care, Columbia University College of Physicians \& Surgeons, Morgan Stanley Children's Hospital of New York,
New York, New York
${ }^{11}$ Department of Pediatrics, Division of Cardiac Intensive Care, University of Wisconsin, Madison, Wisconsin
${ }^{12}$ Department of Pediatrics, Division of Critical Care Medicine, University of lowa Stead Family Children's Hospital, lowa City, lowa
${ }^{13}$ Department of Pediatrics, Division of Cardiac Critical Care, Zucker School of Medicine at Hofstra/Northwell, Hempstead, New York
${ }^{14}$ Cohen Children's Medical Center, New Hyde Park, New York
${ }^{15}$ Department of Biostatistics, Indiana University School of Medicine \& Richard M. Fairbanks School of Public Health, Indianapolis, Indiana
${ }^{16}$ Department of Pediatrics, Division of Critical Care Medicine, University of Utah School of Medicine, Primary Children's Hospital, Salt Lake City, Utah

Correspondence

Christine M. Riley, Department of Pediatrics, Division of Cardiac Critical Care,

Children's National Health System, 111

Michigan Ave, Washington, DC 20010.

Email: criley@childrensnational.org

\section{Funding information}

Funding from the Department of Pediatrics at Indiana University School of Medicine was provided for this study through a Riley

\section{Abstract}

Background: Elevated pulmonary vascular resistance (PVR) is common following repair of truncus arteriosus. Inhaled nitric oxide (iNO) is an effective yet costly therapy that is frequently implemented postoperatively to manage elevated PVR. Objectives: We aimed to describe practice patterns of iNO use in a multicenter cohort of patients who underwent repair of truncus arteriosus, a lesion in which recovery is often complicated by elevated PVR. We also sought to identify patient

Participating Institutions (where work was performed): Riley Hospital for Children, Indianapolis, IN; Cleveland Clinic, Cleveland, OH; Children's Hospital of Michigan, Detroit, MI; Morgan Stanley Children's Hospital of New York, New York, NY; Cohen Children's Medical Center, New Hyde Park, NY; Medical University of South Carolina Children's Hospital, Charleston, SC; Children's National Health System, Washington, DC; Arnold Palmer Hospital for Children, Orlando, FL; Seattle Children's Hospital, Seattle, WA; Ann \& Robert H. Lurie Children's Hospital of Chicago, IL; University of lowa Stead Family Children's Hospital, lowa City, IA; Cincinnati Children's Hospital Medical Center, Cincinnati, OH; Primary Children's Hospital, Salt Lake City, UT; University of Michigan C.S. Mott Children's Hospital, Ann Arbor, MI; American Family Hospital, Madison, WI. 
Children's Foundation Grant (Intramural) for administrative support. No honorarium or other form of payment was provided to anyone to produce the manuscript. and center factors that were more commonly associated with the use of iNO in the postoperative period.

Design: Retrospective cohort study.

Setting: 15 tertiary care pediatric referral centers.

Patients: All infants who underwent definitive repair of truncus arteriosus without aortic arch obstruction between 2009 and 2016.

Interventions: Descriptive statistics were used to demonstrate practice patterns of iNO use. Bivariate comparisons of characteristics of patients who did and did not receive iNO were performed, followed by multivariable mixed logistic regression analysis using backward elimination to identify independent predictors of iNO use.

Main Results: We reviewed 216 patients who met inclusion criteria, of which 102 (46\%) received iNO in the postoperative period: 69 (68\%) had iNO started in the operating room and 33 (32\%) had iNO initiated in the ICU. Median duration of iNO use was 4 days (range: 1-21 days). In multivariable mixed logistic regression analysis, use of deep hypothermic circulatory arrest (odds ratio: 3.2; 95\% confidence interval: 1.2, 8.4) and center (analyzed as a random effect, $p=.02$ ) were independently associated with iNO use.

Conclusions: In this contemporary multicenter study, nearly half of patients who underwent repair of truncus arteriosus received iNO postoperatively. Use of iNO was more dependent on individual center practice rather than patient characteristics. The study suggests a need for collaborative quality initiatives to determine optimal criteria for utilization of this important but expensive therapy.

\section{KEYWORDS}

circulatory arrest, congenital, deep hypothermia induced, heart defects, multicenter study, nitric oxide, postoperative period, truncus arteriosus

\section{1 | INTRODUCTION}

Recovery from surgery for congenital heart disease is frequently complicated by elevated pulmonary vascular resistance (PVR), which can cause clinical decompensation secondary to pulmonary hypertension and right ventricular failure. Inhaled nitric oxide (iNO) is an effective but expensive therapy commonly implemented in this clinical scenario. ${ }^{1-3}$ iNO acts as a selective pulmonary vasodilator by activating soluble guanylate cyclase converting guanosine triphosphate into cyclic guanosine monophosphate. This cascade decreases pulmonary vascular tone without adverse systemic effects. iNO is an effective treatment for potentially life-threatening exacerbations of pulmonary hypertension in the period following cardiopulmonary bypass. $^{4-6}$ It can also be used prophylactically, in hopes of preventing the undesirable hemodynamic effects of elevated PVR such as low cardiac output secondary to right ventricular dysfunction. ${ }^{4}$ Pulmonary hypertension has been associated with longer duration of mechanical ventilation, prolonged ICU stay, hospital stay, and mortality for children undergoing surgery for congenital heart disease. Mitigation of pulmonary hypertension is therefore an important postoperative goal. ${ }^{7,8}$

Truncus arteriosus is a complex congenital cardiac anomaly, first described by Wilson in $1798,{ }^{9}$ wherein the pulmonary arteries fail to separate from the aorta in utero resulting in a common arterial trunk. A large left-to-right shunt often results from this anatomy, preventing PVR from decreasing during the neonatal period and thus predisposing these children to postoperative pulmonary hypertension. This lesion is typically repaired surgically shortly after birth, ${ }^{10,11}$ with many children receiving iNO postoperatively. ${ }^{12-14}$ The indications for iNO use after repair of truncus arteriosus and other congenital heart surgeries are not well defined. We therefore sought to examine the use of iNO following surgical repair of truncus arteriosus, a relatively homogenous group of patients across institutions, to provide insight into contemporary practice patterns surrounding this effective but expensive therapy. We also aimed to identify whether any specific patient or center factors were more likely to be associated with iNO use in the postoperative period.

\section{MATERIALS AND METHODS}

\section{1 | Study population}

We performed secondary analysis of data collected by retrospective review of infants who underwent primary surgical repair of truncus arteriosus between 2009 and 2016 at 15 tertiary care centers within 
the United States. The study was approved by the institutional review boards at all participating centers and the need for informed consent was waived given the retrospective nature of data collection. A list of participating institutions is provided in Supplemental Table 1. The following patients were excluded from analysis: (1) children who underwent pulmonary artery banding but died prior to definitive repair; (2) children with hemitruncus (ie, right pulmonary artery arising from the aorta) or pseudotruncus (ie, pulmonary atresia with major aortopulmonary collaterals); and (3) children who underwent concomitant repair of truncus arteriosus with IAA or aortic arch obstruction (Van Praagh Type A4).

\subsection{Data collection and definitions}

Preoperative, intraoperative, and postoperative data were collected for all patients. Center volume was defined by average cases per year during the study period and categorized as follows:

- Category I: $\leq 1$ case per year

- Category II: $>1 \leq 2$ cases per year

- Category III: $>2 \leq 3$ cases per year

- Category IV: >3 cases per year

Center characteristics also included data collected via a survey of participating centers on unit composition (mixed cardiac and general ICU population vs dedicated $\mathrm{CICU}$ ) and physician training (primarily ICU-trained, primarily cardiology-trained or dual-trained in both cardiology and intensive care). Preoperative ventilation was defined as mechanical ventilation within 24 hours of surgery. Preoperative inotropic support was defined as use of any inotropic infusion within 24 hours of cardiac surgery. Preoperative shock was defined as $\mathrm{pH}$ less than 7.2 or lactate greater than $4 \mathrm{mg} / \mathrm{dL}$ per the Society of Thoracic Surgeons Congenital Heart Surgery Database (STS-CHSD) definition. ${ }^{15}$ Extubation failure was defined as intubation within 72 hours of initial extubation attempt. Operative mortality was defined as mortality occurring before hospital discharge, within 30 days or the index cardiac operation, or in a secondary chronic care facility (or rehabilitation facility) within 180 days following index cardiac operations per STS-CHSD definition.

\section{3 | Statistical analysis}

Data including patterns of iNO use are represented using descriptive statistics. Medians with 25th and 75th percentiles for continuous variables and absolute counts with percentages for categorical variables were employed unless otherwise noted. Bivariate analyses were performed comparing characteristics of patients who received iNO therapy to patients who did not using Wilcoxon sum rank tests and Chi-squared tests. All variables with $P$ values $<.2$ on bivariate analyses were considered for inclusion in a multivariate logistic regression model. Linearity in the logit was examined for continuous variables prior to model building; variables with evidence of nonlinearity were converted to categorical variables. Multivariable logistic regression analysis was performed using stepwise selection to identify independent predictors of iNO use. The multivariable logistical model was then analyzed as a mixed model including center as a random effect. Results of the multivariable analysis are reported as odds ratio (OR) with 95\% confidence intervals (CI). All statistical analyses were performed using STATA version 14 and SAS version 9.4.

\section{3 | RESULTS}

We identified and reviewed 216 patients with truncus arteriosus who met the inclusion criteria. During the study period, 102 (46\%) received iNO therapy in the postoperative period, 69 (68\%) of whom had iNO started in the operating room, and 33 (32\%) of whom had iNO initiated in the ICU following surgery. Median duration of iNO use was 4 days (range: 1-21) and median maximum dose was 20 ppm (range: 10-40). The proportion of patients who received iNO therapy did not change significantly over the duration of study period (Figure 1). The relationship between center and iNO use is illustrated in Figure 2. There was significant variation in iNO use across centers. Additionally, for patients who received iNO, the location where iNO was initiated varied significantly across centers, such that initiation of iNO in the OR was the dominant practice at some centers while initiation of iNO more commonly occurred in the ICU at other centers.

The relationship of center characteristics and iNO use is summarized in Table 1. There was a significant association between center volume and iNO use, with the highest volume centers less likely to implement this therapy, $P<.001$. Use of iNO was also more frequently implemented in centers with multidisciplinary pediatric ICUs (as compared to dedicated cardiac ICUs) and centers at which attending physicians had predominantly ICU training (as compared to centers at which attending physicians had predominantly cardiology training). Centers where the majority of intensivists were dualtrained had the highest proportion of patients who received iNO, though 23 of the 26 patients at these centers who received iNO had the therapy initiated in the OR rather than in the ICU.

Comparisons of baseline or preoperative characteristics of patients who did and did not receive iNO after their definitive truncus arteriosus repair are listed in Table 2. On bivariate analysis, iNO patients tended to be older at the time of their first operation (ie, definitive repair in 213 patients, pulmonary artery banding followed by definitive repair in three patients) and the time from diagnosis to the first operation was significantly longer in patients who received iNO. Patients who received iNO were also significantly more likely to be prescribed furosemide preoperatively and have diminished left ventricular function on preoperative echocardiogram. Bivariate comparison of operative variables is provided in Table 3. From this analysis, duration of cardiopulmonary bypass as well as use of modified ultrafiltration, deep hypothermic circulatory arrest (DHCA), and intraoperative corticosteroids were significantly more common in patients who received iNO postoperatively. 
FIGURE 1 Use of iNO over time among patients who underwent truncus arteriosus repair between 2009 and 2016. The proportion of patients who received iNO (white) as compared to the proportion of patients who did not receive iNO (black) after surgical repair did not vary significantly over time $(p=.44)$
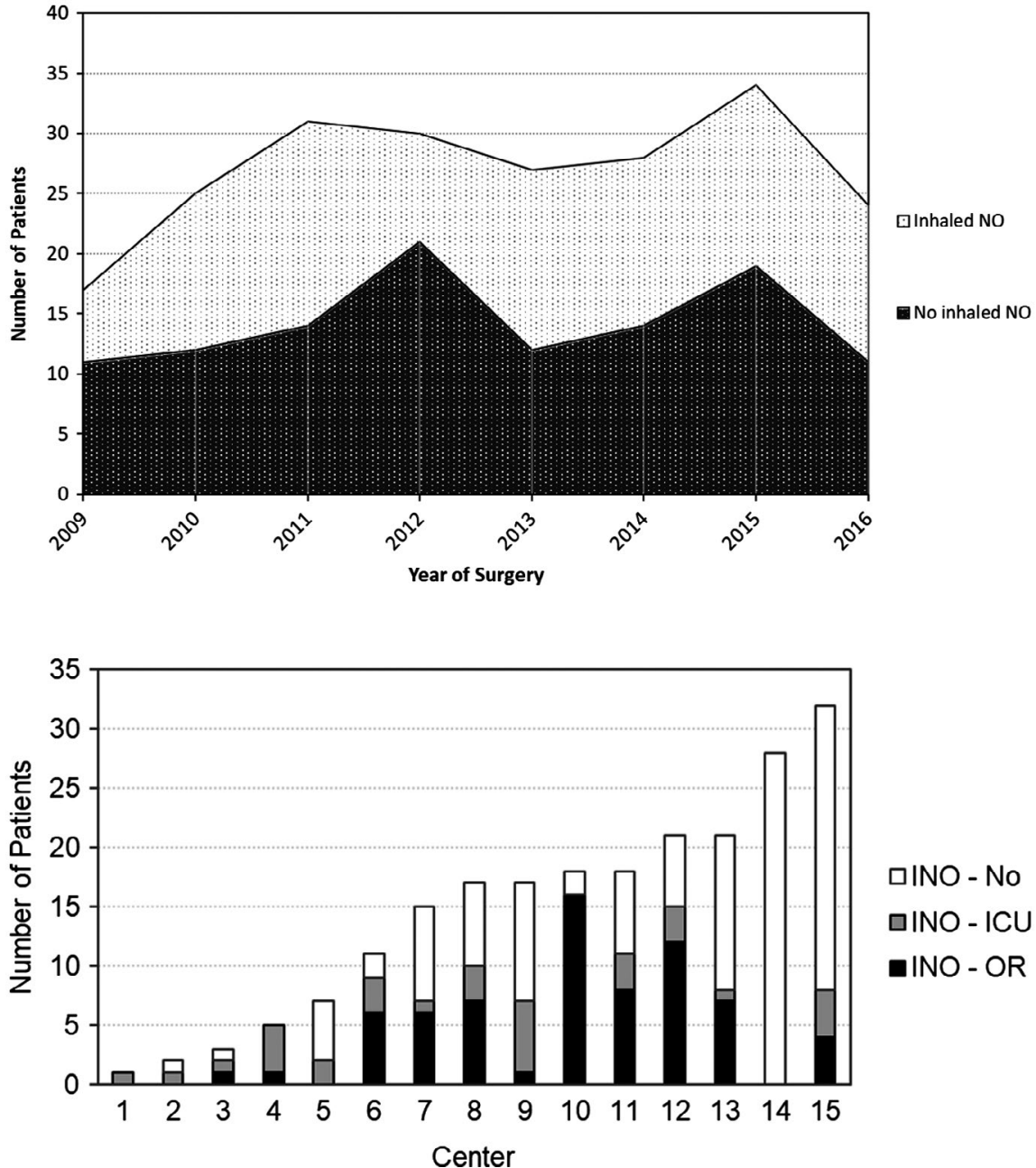

FIGURE 2 Variation in iNO use after definitive repair of truncus arteriosus across centers (2009-2016). Patients who received iNO initiated in the OR (black), patients who received iNO initiated in the ICU, and patients who did not receive iNO (white) are provided for each of the 15 participating centers. The proportion of patients who received iNO $(P<.001)$ and the location where iNO was initiated $(P<$ .001) varied significantly across centers
TABLE 1 Center characteristics for patients who did and did not receive iNO after repair of truncus arteriosus (2009-2016)

\begin{tabular}{|c|c|c|c|c|}
\hline Variable & All patients $(N=216)$ & No iNO $(n=114)$ & $\mathrm{iNO}(n=102)$ & $P$ value \\
\hline Postoperative ICU care model & & & & $<.001$ \\
\hline Dedicated CICU $(n=10)$ & 194 (90\%) & $110(96 \%)$ & $84(82 \%)$ & \\
\hline Multidisciplinary ICU $(n=5)$ & $22(10 \%)$ & $4(4 \%)$ & $18(18 \%)$ & \\
\hline \multicolumn{4}{|c|}{ Predominant training pathway of ICU attending physicians } & .017 \\
\hline ICU training $(n=11)$ & $128(59 \%)$ & $68(60 \%)$ & $60(58 \%)$ & \\
\hline Cardiology training $(n=2)$ & $53(25 \%)$ & $37(32 \%)$ & $16(16 \%)$ & \\
\hline $\begin{array}{l}\text { ICU + Cardiology training } \\
(n=2)\end{array}$ & $35(16 \%)$ & $9(8 \%)$ & $26(25 \%)$ & \\
\hline Center volume & & & & $<.001$ \\
\hline$\leq 1$ surgery/year $(n=5)$ & $18(8 \%)$ & $7(6 \%)$ & $11(11 \%)$ & \\
\hline (1-2] surgeries/year $(n=2)$ & $26(12 \%)$ & $10(9 \%)$ & $16(16 \%)$ & \\
\hline$(2-3]$ surgeries/year $(n=6)$ & $112(52 \%)$ & $45(40 \%)$ & $67(66 \%)$ & \\
\hline$>3$ surgeries/year $(n=2)$ & $60(28 \%)$ & $52(46 \%)$ & $8(8 \%)$ & \\
\hline
\end{tabular}

Results of our multivariable analysis are shown in Table 4. In a model unadjusted for center, use of intraoperative corticosteroids, use of modified ultrafiltration, use of deep hypothermic circulatory arrest, and depressed left ventricular function prior to surgery were identified as independent risk factors for use of iNO postoperatively.
Duration of cardiopulmonary bypass, though not statistically significant, was deemed to have an appreciable effect on the model and therefore also included. When center was added to the model, only use of DHCA (OR: 3.2, 95\% Cl: 1.2, 8.4, $P=.018$ ) and center ( $P=.02)$ was independently associated with iNO. 
TAB LE 2 Preoperative characteristics for patients who did and did not receive iNO therapy after repair of truncus arteriosus (2009-2016)

\begin{tabular}{|c|c|c|c|c|}
\hline Variable $^{a}$ & All patients $(N=216)$ & No iNO $(n=114)$ & $\mathrm{iNO}(n=102)$ & $P$ value \\
\hline Age at diagnosis (days) & $0(0,2)$ & $0.0(0,2)$ & $0(0,1)$ & .64 \\
\hline Van Praagh $1 \mathrm{~A}$ & $112(52 \%)$ & $65(57 \%)$ & $47(46 \%)$ & \\
\hline Van Praagh 1B & 90 (42\%) & $41(36 \%)$ & 49 (48\%) & \\
\hline Female sex & $108(50 \%)$ & $56(49 \%)$ & $52(51 \%)$ & .79 \\
\hline Race & & & & .13 \\
\hline Caucasian & 147 (68\%) & 84 (74\%) & $63(62 \%)$ & \\
\hline African American & $33(15 \%)$ & $16(14 \%)$ & 17 (17\%) & \\
\hline Chromosomal anomaly, any & $83(38 \%)$ & 49 (43\%) & 34 (33\%) & .15 \\
\hline DiGeorge/22q.11 deletion ${ }^{b}$ & $61(28 \%)$ & $36(32 \%)$ & $25(25 \%)$ & .25 \\
\hline Non-cardiac abnormalities & $63(29 \%)$ & $29(25 \%)$ & $34(33 \%)$ & .20 \\
\hline Preoperative shock & $21(10 \%)$ & $9(8 \%)$ & $12(12 \%)$ & .34 \\
\hline Preoperative ventilation ${ }^{c}$ & 45 (21\%) & 22 (19\%) & $23(23 \%)$ & .56 \\
\hline Preoperative furosemide & $146(68 \%)$ & 70 (61\%) & $76(75 \%)$ & .04 \\
\hline Preoperative inotropic support ${ }^{c}$ & $28(13 \%)$ & 15 (13\%) & $13(13 \%)$ & .93 \\
\hline Age at first operation (days) ${ }^{d}$ & $10(7,23)$ & $8.5(6,16)$ & $11.5(7,27)$ & .06 \\
\hline Diagnosis to first operation (days) & $8(5.5,15)$ & $7(5,13)$ & $9(6,20)$ & .04 \\
\hline Moderate & 35 (16\%) & $16(14 \%)$ & 19 (19\%) & \\
\hline Moderate-severe & $9(4 \%)$ & $5(4 \%)$ & $4(4 \%)$ & \\
\hline Severe & $8(4 \%)$ & $2(2 \%)$ & $6(6 \%)$ & \\
\hline
\end{tabular}

${ }^{a}$ Continuous variables presented as median (25\%, 75\%), categorical data presented as counts (\%).

${ }^{\mathrm{b}} 207$ of 216 patients were tested for DiGeorge syndrome/22q.11 deletion.

${ }^{\mathrm{C}}$ Within 24 hours of cardiac surgery.

${ }^{\mathrm{d}}$ Three patients received pulmonary artery banding as a first operation prior to subsequent definitive repair.

Postoperative characteristics and clinical outcomes for patients who did and did not receive iNO are provided in Table 5. Disease acuity was higher in patients who received iNO; significantly greater VVR scores 12 hours postoperatively, more frequent use of ECMO or CPR, and significantly longer durations of mechanical ventilation and hospital stay were observed in this cohort.

We also performed a subanalysis comparing all variables listed in Tables 1-3 and outcomes listed in Table 5 from patients in which iNO was initiated in the OR and patients in which iNO was initated in the ICU. Select variables from this bivariate comparison are provided in Table 6 (supplemental online only). A significantly greater proportion of patients had iNO initiated in the OR at centers with dedicated cardiac ICUs and at centers in which the attending physicians caring for these patients were predominantly ICU trained, while there was no significant difference in center volume (data not shown), exposure to DHCA, or any postoperative outcomes between the two subgroups.

\section{DISCUSSION}

In our analysis of a multicenter cohort of children who underwent repair of truncus arteriosus, we found the utilization of iNO to be fairly common, with nearly half of patients included receiving iNO in 
TABLE 3 Comparison of operative characteristics of patients who did and did not receive iNO therapy after repair of truncus arteriosus (2009-2016)

TAB LE 4 Multivariable mixed logistic regression analysis for predictors of iNO use after repair of truncus arteriosus

\begin{tabular}{lllll}
\hline Variable $^{\text {a }}$ & All patients $(\mathrm{N}=216)$ & No iNO $(n=114)$ & iNO $(n=102)$ & P value \\
\hline CPB duration (min) & $150(124,186)$ & $143(118,179)$ & $158(130,203)$ & .008 \\
\hline CPB less than $150 \mathrm{~min}$ & $108(50 \%)$ & $65(57 \%)$ & $43(42 \%)$ & .03 \\
\hline Cross-clamp duration $(\mathrm{min})$ & $86(73,111)$ & $90(70,111)$ & $85(74,111)$ & .56 \\
\hline DHCA $(n)$ & $31(14 \%)$ & $10(9 \%)$ & $21(21 \%)$ & .01 \\
\hline Lowest temperature $\left({ }^{\circ} \mathrm{C}\right)$ & $25.0(21.4,28.0)$ & $25.4(22.0,28.0)$ & $25(20.0,28.0)$ & .06 \\
\hline MUF use & $139(64 \%)$ & $61(54 \%)$ & $78(77 \%)$ & $<.001$ \\
\hline $\begin{array}{l}\text { Intraoperative } \\
\text { corticosteroids }\end{array}$ & $161(75 \%)$ & $76(67 \%)$ & $85(83 \%)$ & .005 \\
\hline RV-PA conduit size $(\mathrm{mm})$ & $11(9,12)$ & $11(9,12)$ & $11(9,12)$ & .97 \\
\hline $\begin{array}{l}\text { RV-PA conduit size }(\mathrm{mm} / \\
\left.\mathrm{m}^{2}\right)\end{array}$ & $51(46,56)$ & $51(46,57)$ & $51(45,56)$ & .49 \\
\hline $\begin{array}{l}\text { Truncal valve surgery } \\
\text { Factor VIla }\end{array}$ & $37(17 \%)$ & $19(17 \%)$ & $18(18 \%)$ & .85 \\
\hline \hline
\end{tabular}

Abbreviations: CPB, cardiopulmonary bypass; DHCA, deep hypothermic circulatory arrest; MUF, modified ultrafiltration; RV-PA, right ventricle-to-pulmonary artery.

${ }^{a}$ Continuous variables presented as median (25\%, $\left.75 \%\right)$, categorical variables presented as counts (\%).

\begin{tabular}{llll} 
Variable & Odds ratio & 95\% confidence interval & P value \\
\hline Unadjusted model & & & \\
\hline Modified ultrafiltration & 3.8 & $2.0-7.2$ & $<.001$ \\
\hline Intraoperative corticosteroids & 4.0 & $1.9-8.4$ & $<.001$ \\
\hline Depressed left ventricular function & 4.4 & $1.5-13.0$ & .008 \\
\hline Deep hypothermic circulatory arrest & 2.9 & $1.2-7.2$ & .017 \\
\hline Cardiopulmonary bypass $>150$ min & 1.8 & $1.0-3.2$ & .067 \\
\hline Model adjusted for center & & & .034 \\
\hline Deep hypothermic circulatory arrest & 2.9 & $1.1-7.5$ & .02 \\
\hline Center & & & \\
\hline
\end{tabular}

${ }^{a}$ Analyzed in the model as a random effect; odds ratios for individual centers were not calculated.

\begin{tabular}{llllc} 
Variable $^{\text {a }}$ & All patients $(\mathrm{N}=216)$ & No iNO $(n=114)$ & iNO $(n=102)$ & P value \\
\hline Delayed sternal closure & $126(58 \%)$ & $64(56 \%)$ & $62(61 \%)$ & .49 \\
\hline ECMO & $22(10 \%)$ & $6(5 \%)$ & $16(6 \%)$ & .01 \\
\hline CPR & $26(12 \%)$ & $8(7 \%)$ & $18(18 \%)$ & .02 \\
\hline Reoperation for bleeding & $19(9 \%)$ & $7(6 \%)$ & $12(12 \%)$ & .15 \\
\hline $\begin{array}{l}\text { Reoperation not for } \\
\text { bleeding }\end{array}$ & $25(12 \%)$ & $9(8 \%)$ & $16(16 \%)$ & .07 \\
\hline $\begin{array}{l}\text { VVR at } 12 h^{b} \\
\text { Mechanical ventilation (h) }\end{array}$ & $140(86,264)$ & $108(72,168)$ & $193(116,532)$ & $<.001$ \\
\hline Extubation failure & $22(10 \%)$ & $9(8 \%)$ & $13(13 \%)$ & .24 \\
\hline Hospital LOS (days) & $23(15,43)$ & $20(13,33)$ & $28(18,51)$ & $<.001$ \\
\hline Operative mortality & $15(7 \%)$ & $5(4 \%)$ & $10(10 \%)$ & .12 \\
\hline
\end{tabular}

Abbreviations: CPR, cardiopulmonary resuscitation; ECMO, extracorporeal membrane oxygenation; LOS, length-of-stay.

${ }^{a}$ Continuous variables presented as median (25\%, 75\%), categorical variables presented as counts (\%). ${ }^{b}$ Vasoactive-ventilation-renal score $=$ vasoactive-inotrope score + ventilation index $+\Delta$ creatinine [change in serum creatinine from baseline*10], not calculated for 17 patients on ECMO at 12 hours. 
TAB LE 6 Comparison of characteristics and outcomes of patients who had inhaled nitric oxide (iNO) initiated in the operating room (OR) versus the ICU

\begin{tabular}{|c|c|c|c|}
\hline Variable & $\begin{array}{l}\text { iNO in OR } \\
(n=69)\end{array}$ & $\begin{array}{l}\text { iNO in ICU } \\
(n=33)\end{array}$ & $P$ value \\
\hline Prenatal diagnosis & 38 (55\%) & 25 (76\%) & .04 \\
\hline \multicolumn{3}{|l|}{ Truncus type } & \multirow[t]{4}{*}{.04} \\
\hline Van Praagh $1 \mathrm{~A}$ & $26(38 \%)$ & $21(64 \%)$ & \\
\hline Van Praagh 1B & 39 (57\%) & $10(30 \%)$ & \\
\hline Van Praagh $1 \mathrm{C}$ & $4(6 \%)$ & $26 \%)$ & \\
\hline $\begin{array}{l}\text { No diagnosis before } \\
\text { nursery discharge }\end{array}$ & $16(23 \%)$ & $5(15 \%)$ & .44 \\
\hline $\begin{array}{l}\text { Age at first operation } \\
\text { (days) }\end{array}$ & $11(7,30)$ & $12(8,20)$ & .81 \\
\hline $\begin{array}{l}\text { Preoperative decreased } \\
\text { RV function }\end{array}$ & $14(20 \%)$ & $5(15 \%)$ & .60 \\
\hline $\begin{array}{l}\geq \text { moderate preopera- } \\
\text { tive valve insufficiency }\end{array}$ & $18(26 \%)$ & $11(33 \%)$ & .45 \\
\hline $\begin{array}{l}\text { Cardiopulmonary by- } \\
\text { pass duration }\end{array}$ & $\begin{array}{l}158(128, \\
201)\end{array}$ & $\begin{array}{l}159(135 \\
214)\end{array}$ & .85 \\
\hline $\begin{array}{l}\text { Deep hypothermic } \\
\text { circulatory arrest }\end{array}$ & $16(23 \%)$ & $5(15 \%)$ & .35 \\
\hline Lowest temperature $\left({ }^{\circ} \mathrm{C}\right)$ & $24(20,28)$ & $25(24,28)$ & .22 \\
\hline $\begin{array}{l}\text { Modified ultrafiltration } \\
\text { use }\end{array}$ & $56(81 \%)$ & $22(67 \%)$ & .10 \\
\hline $\begin{array}{l}\text { Intraoperative } \\
\text { corticosteroids }\end{array}$ & $61(88 \%)$ & $24(72 \%)$ & .047 \\
\hline $\begin{array}{l}\text { Concomitant truncal } \\
\text { valve surgery }\end{array}$ & $10(14 \%)$ & $8(24 \%)$ & .23 \\
\hline $\begin{array}{l}\text { Recovered in dedicated } \\
\text { cardiac ICU }\end{array}$ & 61 (88\%) & $23(70 \%)$ & .02 \\
\hline \multicolumn{3}{|c|}{ Training pathway of ICU physicians } & .02 \\
\hline $\mathrm{ICU}$ & 35 (51\%) & $25(76 \%)$ & \\
\hline Cardiology & $11(16 \%)$ & 5 (15\%) & \\
\hline ICU + Cardiology & $23(33 \%)$ & $3(9 \%)$ & \\
\hline Delayed sternal closure & 44 (64\%) & $18(55 \%)$ & .37 \\
\hline Postoperative ECMO & 11 (16\%) & 5 (15\%) & 1.00 \\
\hline Postoperative CPR & 9 (13\%) & 9 (27\%) & .08 \\
\hline $\begin{array}{l}\text { Unplanned reoperation } \\
\text { or catheterization }\end{array}$ & 23 & 11 & 1.00 \\
\hline $\begin{array}{l}\text { Mechanical ventilation } \\
\text { (hours) }\end{array}$ & $\begin{array}{l}169(100 \\
339)\end{array}$ & $\begin{array}{l}168(122 \\
216)\end{array}$ & .60 \\
\hline Hospital LOS (days) & $30(15,51)$ & $25(18,49)$ & .99 \\
\hline Operative mortality & $6(9 \%)$ & $4(12 \%)$ & .73 \\
\hline
\end{tabular}

Continuous variables represented as median (25\%,75\%), categorical data represented as counts (\%).

the postoperative period. While the percentage of patients who received iNO postoperatively remained relatively consistent over time, we noted considerable variation in iNO practice patterns across centers and determined center to be independently associated with postoperative iNO therapy. We assume that some of the patients who received iNO likely did so in response to clinical evidence of potentially life-threatening right ventricular hypertension, but the observed variability in usage across centers, with some centers initiating iNO in all or most of their patients and other centers utilizing the therapy in few or none of their patients, suggests that iNO utilization in some patients may not have been necessary but rather driven by center practice.

We did identify associations between some center characteristics and iNO utilization. For example, lower volume centers tended to use iNO more frequently, a finding that was also noted in a prior study examining use of iNO in cardiac centers participating in the Pediatric Health Information System database. ${ }^{16} \mathrm{We}$ also noted an increase in iNO use in centers without dedicated cardiac ICUs or centers where patients were managed by physicians with critical care training. While it is possible that providers, either in the OR and in the ICU, who less commonly encounter children with this complex lesion may have lower thresholds to start iNO as a precautionary measure, or that iNO use could be influenced by ICU care model or physician training, we cannot make definitive conclusions about these relationships based on the limited number of centers in each of the designated categories. It must also be noted that there are undoubtedly intangible factors that influenced providers' decision whether or not to initiate iNO that could not be measured in this study, some of which are likely to be center-specific.

Variability of iNO usage is not unique to patients with congenital heart disease. Other studies have found similar patterns of variability in other patient populations including premature infants and infants following congenital diaphragmatic hernia repair. ${ }^{17,18}$ Moreover, in a study of neonates with persistent pulmonary hypertension, an effort to protocolize initiation and weaning of iNO was shown to be successful in decreasing utilization and, accordingly, the costs associated with this therapy. ${ }^{19}$ To our knowledge, however, effective protocols identifying clinical triggers for initiation and weaning of iNO following pediatric cardiac surgery are generally lacking in the literature. Simsic and coworkers described their attempt to decrease iNO usage and minimize practice variation using an initiation protocol for pediatric patients with cardiac disease. ${ }^{20}$ This study found that while variation among providers was reduced, usage rates and associated costs did not change, even with generally high compliance to the protocol. In another single center study by Tzanetos et al, the authors were able to demonstrate a decrease in direct costs associated with iNO use following implementation of an iNO initiation and weaning protocol in a mixed cardiac and general pediatric intensive care unit, though protocol adherence did not correlate with the decrease in cost. ${ }^{21}$ In these studies, protocols were aimed at reducing variation across providers within a single institution.

Given the marked variation in iNO use across centers observed in our study, multicenter collaboration to create protocols aimed at reducing variation in iNO use across institutions could be more illuminating and should be pursued. In particular, collaboration between centers with very low and very high iNO utilization rates could identify potential pathways for decreasing iNO use at the 
latter institutions. Further, protocols containing physiologic triggers for initiation, whether it be in the operating room or ICU, with goal-driven maintenance algorithms are reasonable starting points. Resource utilization and associated costs of caring for children with congenital heart disease remain high. Using data from the Pediatric Health Information System database, Smith and colleagues found preoperative and postoperative management charges for patients undergoing congenital heart surgery in 2011 to be approximately $\$ 433875$ per case. ${ }^{22}$ The authors also found that mean duration of iNO use escalated from 2005 to 2011 (ie, 5.3 days to 6.6 days) and represented more than $\$ 50000$ of the average charges per patient exposed. Thus, as healthcare costs continue to rise, protocols to guide iNO usage may represent an opportunity for resource conservation and cost containment. To reduce the marked variation of iNO use we observed, utilization of a multicenter quality improvement collaborative to create and implement a standardized protocol for iNO initiation, including assessment of patient response to therapy and deliberate weaning if no objective evidence of clinical benefit are apparent, would be a reasonable next step.

In addition to center, we identified DHCA to be an independent predictor of iNO usage. From our data, however, we are unable to definitively discern whether the latter finding reflects a physiological relationship between DHCA and elevated PVR leading to the use of iNO to mitigate pulmonary hypertension or right ventricular dysfunction, or a heightened perception of patient acuity by providers in the setting of DHCA prompting prophylactic iNO use. DHCA could prolong the necessity for cardiopulmonary bypass due to rewarming, and this prolonged exposure to cardiopulmonary bypass could result in more exaggerated elevations in PVR postoperatively. In a seminal report by Wernovsky et al, elevated PVR was observed in patients who underwent the arterial switch operation with DHCA or low-flow cardiopulmonary bypass. ${ }^{23}$ Additionally, in a porcine model of hypothermic circulatory arrest, Cooper and colleagues demonstrated impaired endothelial vasorelaxation in cerebral arteries, renal arteries, and pulmonary veins. ${ }^{24}$ Based on these data and the center-independent association between DHCA and iNO use, it is plausible that the observed relationship between DHCA and iNO use was related to pathologic increase in PVR. Exposure to DHCA may therefore be an appropriate clinical trigger for iNO usage after surgery for congenital heart disease.

This study has several limitations. The retrospective nature of the study affected our ability to include a more exhaustive list of clinically relevant variables in our analyses. Most notably, specific clinical or echocardiography findings commonly associated with elevated PVR (eg, direct measurements of pulmonary artery pressures; tricuspid valve regurgitation jet velocity) were not routinely captured by most centers, preventing us from being able to assess the clinical decision-making process behind initiation of iNO. The sparsity of this information in the medical record, however, suggests that the decision to start iNO in many cases was likely not based on objective data but rather was prophylactic or based on subjective concerns for right ventricular hypertension. Other treatment strategies that may have mitigated the presence or severity of pulmonary hypertension, such as acid-base management, use of other pulmonary vasodilatory therapies, or the use of pharmacologic paralysis, were not assessed. The study was also not designed to determine if iNO usage had a significant effect on patient outcomes. The associations identified between iNO use and worse clinical outcomes should therefore not be interpreted as causal. Based on the marked variation in iNO use across centers, however, a study that randomizes patients with moderate postoperative disease severity to empirically receive or not receive iNO therapy could be possible and should be pursued.

\section{5 | CONCLUSIONS}

In a contemporary multicenter dataset, nearly half of patients with truncus arteriosus who underwent repair received $\mathrm{iNO}$, and usage did not vary significantly over time. We identified DHCA as an independent risk factor for iNO use and observed a strong relationship between center and iNO use while adjusting for patient characteristics suggesting center practice as an important predictor of iNO use. These findings indicate a need for multicenter collaborative quality improvement initiatives to determine best practices for this important but expensive therapy.

\section{ACKNOWLEDGMENTS}

We like to acknowledge and thank the following individuals who assisted the authors with administrative tasks, data collection, or review of echocardiograms for this study: Amy Moravec, BS, Ann \& Robert H. Lurie Children's Hospital of Chicago; Kari Nelson, BA, MSN, CPNP, University of Wisconsin-American Family Children's Hospital; Josh Belfer MD, Resident in Pediatrics, Cohen Children's Medical Center of New York; Eva Cheung MD, Assistant Professor, Department of Pediatrics, Division of Cardiology, Columbia University College of Physicians \& Surgeons, Morgan Stanley Children's Hospital of New York, New York, NY; Linda M. Lambert MSN-cFNP, Administrative Director of Research, Heart CenterPrimary Children's Hospital, University of Utah; Lisa Michelle Hansen, Clinical Research Coordinator, Heart Center-Primary Children's Hospital, University of Utah.

\section{CONFLICT OF INTEREST}

None of the authors have conflicts of interest to disclose.

\section{AUTHOR CONTRIBUTIONS}

Critique of study protocol, data collection, drafting and revision of manuscript: Riley

Study concept and design, data collection, data analysis and interpretation, critical revision, approval of article: Mastropietro

Critique of study protocol, data collection, approval of article: Sassalos Critique of study protocol, data collection, critical revision, approval of article: Buckley, Costello, Iliopoulos, Jennings, Cashen, Suguna Narasimhulu, Gowda, Smerling, Wilhelm, Badheka, Bakar 
Study design, data analysis, statistic analysis, approval of article: Moser Critique of study protocol, data collection, data interpretation, critical revision, approval of article: Amula

\section{ORCID}

Christine M. Riley (iD) https://orcid.org/0000-0003-2224-0649

Christopher W. Mastropietro (iD https://orcid.

org/0000-0002-7409-7664

\section{REFERENCES}

1. Checchia PA, Bronicki RA, Goldstein B. Review of inhaled nitric oxide in the pediatric cardia surgery setting. Pediatr Cardiol. 2012;33(4):493-505. https://doi.org/10.1007/s00246-012-0172-4.

2. Barr FE, Macrae D. Inhaled nitric oxide and related therapies. Pediatr Crit Care Med. 2010;11(2 Suppl):S30-S36. https://doi.org/10.1097/ PCC.0b013e3181c76b42.

3. Taylor MB, Laussen P. Fundementals of management of acute postoperative pulmonary hypertension. Pediatr Crit Care Med. 2010;11(2 Suppl):S27-S29. https://doi.org/10.1097/PCC.0b013e3181c769a4.

4. James C, Millar J, Horton S, et al. Nitric oxide administration during paediatric cardiopulmonary bypass: a randomized controlled trial. Intensive Care Med. 2016;42(11):1744-1752. Epub 30 September 2016.

5. Gorenflo M, Gu H, Xu Z. Peri-operative pulmonary hypertension in paediatric patients: current strategies in children with congenital heart disease. Cardiology. 2010;116:10-17. https://doi. org/10.1159/000313864.

6. Muhiudeen Russell IA, Zwass MS, Fineman JR, et al. The effect of inhaled nitric oxide on postoperative pulmonary hypertension in infant and children undergoing surgical repair of congenital heart diease. Anesth Analg. 1998;87:46-51.

7. Bando K, Turrentine MW, Sharp TG, et al. Pulmonary hypertension after operations for congenital heart diease: analysis of risk factor and management. J Thorac Cardiovasc Surg. 1996;112(6):1600-1609.

8. Bizzarro M, Gross I, Barbosa FT. Inhaled nitric oxide for the postoperative management of pulmonary hypertension in infants and children with congenital heart disease. Cochrane Database Syst Rev. 2014;7:CD005055.

9. Wilson J. A description of a very unusual malformation of the human heart. Phil Trans R Soc London (Biol). 1798;18:346.

10. Thompson LD, McElhinney DB, Reddy VM, Petrossian ED, Silverman $\mathrm{NH}$, Hanley FL. Neonatal repair of truncus arteriosus: continuing improvement in outcomes. Ann Thorac Surg. 2001;72:391-395.

11. Kalavrouziotis G, Purohit M, Ciotti G, Corno AF, Pozzi M. Truncus arteriosus communis: early and midterm results of early primary repair. Ann Thorac Surg. 2006;82:2200-2206.

12. Sandrio S, Ruffer A, Purbojo A, et al. Common arteria trunk: current implementation of the primary and staged repair strategies. Interact Cardiovasc Thorac Surg. 2015;21:754-760.
13. Shamszad P, Moore RA, Ghanayem N, Cooper DS. Intensive care management of neonates with d-transposition of the great arteries and common arterial trunk. Cardiol Young. 2012;22(6):755-760.

14. Nichols DG, Ungerleider RM, Spevak PJ, et al., eds. Critical Heart Disease in Infants and Children. 2nd ed. Philadelphia, PA: Mosby; 2006.

15. Mavroudis C, Jacobs JP, Siegel A, et al. Procedure-based complications to guide informed consent: analysis of society of thoracic surgeons-congenital heart surgery database. Ann Thorac Surg. 2014;97:1838-1849.

16. Gupta P, Rettiganti M, Wilcox A, Eble BK, Schmitz ML, Zakaria D. Spectrum of off-label nitric oxide utilization after pediatric cardiac surgery among centers of varying surgical volume. J Intensive Care Med. 2017 Jan. https://doi.org/10.1177/0885066617728494.

17. Putnam LR, Tsao K, Morini F, et al. Evaluation of variability in inhaled nitric oxide use and pulmonary hypertension in patients with congenital diaphragmatic hernia. JAMA Pediatr. 2016;170(12):1188-1194.

18. Stenger MR, Slaughter JL, Kelleher K, et al. Hospital variation in nitric oxide use for premature infants. Pediatrics. 2012;129(4):e945 -e951.

19. Hughes Driscoll CA, Davis NL, Miles M, El-Metwally D. A quality improvement project to improve evidence-based inhaled nitric oxide use. Respir Care. 2018;63(1):20-27.

20. Simsic JM, Harrison S, Evans $L$, et al. Reducing variation in the use of nitric oxide. Pediatrics. 2014;133(6):e1753-e1758.

21. Todd Tzanetos DR, Housley JJ, Barr FE, May WL, Landers CD. Implementation of an inhaled nitric oxide protocol decreases direct cost associated with its use. Respir Care. 2015;60(5):644-650.

22. Smith AH, Gay JC, Patel NR. Trending in resource utilization associated with the inpatient treatment of neonatal congenital heart disease. Congenit Heart Dis. 2013;9:96-105.

23. Wernovsky G, Wypij D, Jonas RA, et al. Postoperative course and hemodynamic profile after the arterial switch operation in neonates and infants. A comparison of low-flow cardiopulmonary bypass and circulatory arrest. Circulation. 1995;92(8):2226-2235.

24. Cooper WA, Duarte IG, Thourani VH, et al. Hypothermic circulatory arrest causes multisystem vascular endothelial dysfunction and apoptosis. Ann Thorac Surg. 2000;69:696-702.

\section{SUPPORTING INFORMATION}

Additional supporting information may be found online in the Supporting Information section.

How to cite this article: Riley CM, Mastropietro CW, Sassalos $P$, et al. Utilization of inhaled nitric oxide after surgical repair of truncus arteriosus: A multicenter analysis. Congenital Heart Disease. 2019;14:1078-1086. https://doi.org/10.1111/chd.12849 